\title{
NEAR-INFRARED SPECTRA OF COMETS BENNETT AND KOHOUTEK
}

\author{
A. E. Potter, T Morgan, B. Ulrich and T Barnes
}

\begin{abstract}
Spectra in the range 0.9 to 1.6 microns of Comets Bennett and Kohoutek were obtained at the Coudé focus of the 2.72 meter telescope of McDonald Observatory. Comet Bennett was observed on a number of nights in March and April 1970, while spectra of Comet Kohoutek were taken on two nights in December 1973. The spectrum of Comet Bennett showed a strong continuum, which changed markedly in character during the observations. The surface brightness of Comet Kohoutek was less than the terrestrial hydroxyl airglow. The continuum spectrum could not be distinguished beneath the airglow bands. Spectra of both comets displayed clearly the (0-0) transition of the CN red system at $9115 \mathrm{~cm}^{1}$, with indications of the $(0-1) \mathrm{CN}$ transition visible at the edge of a region of strong atmospheric absorption. Several other possible emission lines were noted in the comet spectra. These were all weak, with signal levels marginally greater than the noise. None were positively identified.
\end{abstract}

